\title{
Ciudades intermedias y gobiernos locales: desfases escalares en la Región de La Araucanía, Chile
}

Gonzalo Salazar. Pontificia Universidad Católica de Chile, Santiago, Chile. Felipe Irarrázaval. Pontificia Universidad Católica de Chile, Santiago, Chile. Martín Fonck. Pontificia Universidad Católica de Chile, Santiago, Chile.

RESUMEN | Las ciudades intermedias se caracterizan por una gran cantidad de interacciones con su territorio circundante y con otras ciudades, lo que implica un desafío para sus gobiernos locales. El presente trabajo aborda estos desafíos desde los procesos de intermediación y en las distintas escalas que generan las prácticas espaciales. La metodología empleada es mixta, con el trabajo estructurado a partir del enfoque cualitativo de metodologías móviles, complementado con un análisis de conmutaciones intercomunales. Los resultados dan cuenta de que en los principales nodos de intermediación de la región, los gobiernos locales enfrentan importantes desafíos derivados de los procesos mismos de intermediación, específicamente en materia de vialidad y crecimiento urbano, a la vez que subestiman la relevancia de las dinámicas interculturales en el territorio. Se concluye que abordar estos elementos es fundamental para comprender los procesos escalares que enfrentan los gobiernos locales, para así poder planificar en dirección a la sustentabilidad local.

PALABRAS CLAVE | gobierno local, ciudades intermedias, sustentabilidad urbana.

ABSTRACT | Local governments of intermediate cities face significant challenges regarding their interactions with surrounding areas, as well as with other cities. The present article deals with such challenges, based on two conceptual frameworks: intermediation processes, and scale. The methodological focus utilizes a qualitative approach, based on mobile methodologies, complemented by a quantitative commuting assessment. The results show that local governments from the main intermediation nodes in the Region of La Araucania face problems related with intermediation process, specifically regarding urban sprawl and roadways. Furthermore, local governments underestimate indigenous dynamics within and between cities, which is a key element in the region. The results allow us to conclude that scalar gaps represent a key problem for local governments of intermediate cities, which must be resolved in order to improve planning processes and enhance the local sustainability of such cities.

KEYWORDs | local government, intermediate cities, urban sustainability.

Recibido el 27 de noviembre de 2015, aprobado el 7 de junio de 2016

E-mails: G. Salazar, gonzalosalazar@uc.cl|F. Irarrázaval, firarrazava@uc.cl | M. Fonck, mfonck1@uc.cl 


\section{Introducción}

La última propuesta de la agenda de descentralización en Chile (Comisión Asesora Presidencial en Descentralización y Desarrollo Regional, 2014) reflotó el debate respecto a la organización subnacional del país. Dicha propuesta se centró fundamentalmente en la escala regional y provincial, con el nivel comunal escuetamente considerado. Previamente, lo más cercano a esta escala en la agenda política había sido la definición de nuevas comunas, producto del crecimiento demográfico de ciudades distintas a las capitales comunales (Subsecretaría de Desarrollo Regional [Subdere], 2007). No obstante, más allá de tales aproximaciones, en el plano local se dan procesos que trascienden los límites político-administrativo de las comunas -por ejemplo, los derivados de procesos de movilidad-, los cuales implican importantes desafíos para la labor de los municipios y requieren ser analizados en materia de políticas públicas.

Un posible ejemplo de esto último es lo que acontece en los gobiernos locales asociados a ciudades intermedias, las que se caracterizan por una gran cantidad de interacciones con su territorio circundante, así como con otras ciudades (Bellet, 2009). En tanto los instrumentos y atribuciones municipales en Chile son sumamente estandarizados, los desafíos de los gobiernos locales de ciudades intermedias en materia de planificación urbana son complejos. Este artículo aborda la relación entre los procesos espaciales que se dan en las ciudades intermedias de la Región de la Araucanía y los gobiernos locales, fundamentalmente en materia de planificación. En ese contexto, se desarrollan dos argumentos: primero, que la definición escalar de los gobiernos locales en las ciudades intermedias no logra dar cuenta de las prácticas sociales que acontecen en el espacio; y segundo, que este desfase escalar entre el gobierno local y procesos espaciales genera diversas problemáticas que obstaculizan el desarrollo de las funciones por parte de dichos gobiernos.

Para analizar esta problemática, se vinculan dos ejes conceptuales. Por un lado, se propone el concepto de escala, el cual, mediante las nociones de tamaño, nivel y relación (Howitt, 1998), entrega herramientas para analizar críticamente la creación de divisiones político-administrativas desde las prácticas sociales en el espacio (Kaiser \& Nikiforova, 2008; Moore, 2008). Por otro lado, y fundamentalmente debido al contexto de las ciudades intermedias, se desarrolla la noción de intermediación, que entrega un soporte conceptual para analizar nodos urbanos que interactúan muy activamente con su territorio circundante, y también con otros nodos urbanos (Bellet \& Llop, 2004). Este marco teórico plantea dos requerimientos metodológicos: centrarse en los discursos y prácticas de los actores para identificar las escalas; y examinar las interacciones territoriales de los habitantes de forma móvil, para de ese modo analizar la intermediación. En ese contexto, si bien se opta por una metodología mixta, es la aproximación cualitativa la que guía el trabajo. Además de un extenso trabajo de entrevistas y observación participante, se emplearon metodologías móviles para comprender cómo los discursos explicaban prácticas espaciales (Evans \& Jones, 2011; Anderson, 2004), lo que fue complementado con un análisis de conmutaciones funcionales (Lufin \& Atienza, 2015). 
Desde el punto de vista práctico se espera visibilizar una problemática reconocida por los gobiernos locales de ciudades intermedias, esto es, cómo los procesos de intermediación tienden a dificultar aquellos relacionados con la planificación y gestión local. Atenuar este desfase escalar es fundamental para avanzar hacia ciudades intermedias cuyas administraciones cuenten con mejores atribuciones para gestionarlas desde lo local y con miras a la sustentabilidad. En el aspecto teórico, se espera contribuir a los estudios urbanos mediante la vinculación de dos aspectos claves en las ciudades intermedias, la escala y la intermediación, y a través de la aplicación de estos conceptos a los procesos de planificación en los gobiernos locales.

El artículo se estructura en seis apartados, además de la introducción. Los apartados segundo y tercero presentan las discusiones teóricas en la materia, donde el primero vincula la discusión de los gobiernos locales con la escala, y el segundo desarrolla la noción de intermediación y su relación con los gobiernos locales. El apartado tercero explica brevemente la metodología de trabajo, mientras que los apartados cuarto y quinto dan cuenta de los resultados. En primera instancia se muestran las dinámicas de intermediación mediante conmutaciones intercomunales en la Región de la Araucanía, lo cual es complementado con el discurso de distintos actores al respecto. En segunda instancia son abordadas las problemáticas de los gobiernos locales frente a los procesos de intermediación, siendo las principales aquellas referidas a la vialidad, el crecimiento urbano y la interculturalidad. Finalmente, se desarrollan las conclusiones.

\section{Gobiernos locales y escala: buscando la compresión espacio-temporal}

En la actualidad, el debate respecto a gobiernos locales tiende a ser contextualizado en el marco de la discusión sobre la descentralización territorial (Boisier, 2010; Falleti, 2010; Montecinos, 2005; Programa de Naciones Unidas para el Desarrollo [PNUD], 2007). En términos concretos, la descentralización es definida como un "conjunto de políticas, reformas electorales o reformas constitucionales que transfieren responsabilidades, recursos o autoridad desde niveles de gobierno más altos a niveles más bajos" (Falleti, 2010, p. 34). Es en ese marco donde se debaten los futuros avances en materia regional y comunal en Chile (Comisión Asesora Presidencial en Descentralización y Desarrollo Regional, 2014), al igual que en América Latina y en otros países del mundo (PNud, 2007; Shah, 2006).

Una crítica que se le puede hacer al enfoque de la descentralización es que carece de una discusión respecto al destino espacial de sus propuestas y acciones descentralizadoras. Es decir, le asigna poca o nula relevancia a la división espacial sobre la que va a gobernar la administración local. Ello gatilla una serie de problemáticas en materia de gestión municipal, en la medida en que la delimitación espacial sobre la que deben ejercer no da cuenta de las dinámicas sociales que ocurren en el territorio. No se trata solo de debatir atribuciones, representantes o recursos para gobiernos locales, sino también de reflexionar sobre las formas y procesos espaciales en que decantará la descentralización. 
El esquema conceptual en que se ha desarrollado el debate sobre la descentralización en Chile es ilustrativo de la problemática señalada, en tanto se centra en la transferencia de responsabilidades desde la escala nacional hacia escalas subnacionales, dividida en tres dimensiones principales: política, administrativa y fiscal (Boisier, 2010; Falleti, 2010; PNUD, 2007). Si bien estas tres dimensiones son fundamentales para el ejercicio de los gobiernos locales, el debate sobre la descentralización pareciera darse sobre una ontología espacial escasamente discutida, considerando que no hay mayor reflexión respecto a cómo se definen las unidades administrativas sobre las cuales se va a descentralizar. La ontología espacial que sirve de base al debate sobre la descentralización debiera considerar, además, que la producción social del espacio varía en el tiempo, generando distintas compresiones espacio-temporales en función de las prácticas sociales desplegadas en el espacio en un momento determinado (Massey, 1994).

Este ejercicio conceptual de la compresión espacio-temporal merece ser aplicado en materia de gobiernos locales. Trabajos como el de Berdegué et al. (2011) sobre territorios funcionales, de Vial (2015) respecto a conurbaciones intercomunales, o de Heinrichs, Nuissl y Rodríguez (2009), Fuentes, Allard y Orellana (2007) y Orellana, Allard, Néspolo y Mercado (2012) en relación con gobiernos metropolitanos, dan cuenta de las diversas problemáticas que enfrentan los gobiernos locales frente a las dinámicas espaciales que tienen que administrar y/o gobernar. La crítica de estos trabajos se centra especialmente en que los gobiernos locales no cuentan con las atribuciones necesarias para tratar las problemáticas que se viven en el territorio, lo cual en cierto modo es un efecto de la escasa reflexión dada en las políticas públicas de descentralización a materias como la delimitación de unidades administrativas. Esto se puede interpretar, siguiendo la compresión espacio-temporal de Massey (1994), como un desfase entre las unidades administrativas que se definen y las prácticas sociales que se producen el espacio, en tanto las prácticas espaciales son las que van a significar lo local y desde donde se busca organizar políticamente el gobierno local.

Desde el punto de vista teórico, en el debate sobre escala se puede encontrar una gran cantidad de contribuciones para abordar la problemática de la "compresión espacio-temporal” y su articulación con los gobiernos locales (véase, por ejemplo, Brenner, 2001; Kaiser \& Nikiforova, 2008; Marston, Jones \& Woordward, 2005; Moore, 2008). La escala es una diferenciación fundamental del espacio geográfico, o más precisamente una abstracción de este, y corresponde a un ejercicio analítico destinado a delimitar espacialmente un fenómeno social particular (Smith, 1992). En este ejercicio se dan tres elementos combinados que son fundamentales para definir la escala como tal: tamaño, nivel y relación (Howitt, 1998).

En primer lugar se encuentra el tamaño, que alude a la extensión espacial de la escala, la cual encuentra su correlato en las posibles delimitaciones espaciales que tiene la unidad territorial en cuestión. En segundo lugar se encuentra el nivel, el cual representa el grado de complejidad de las relaciones entre escalas, o bien la jerarquía entre ellas; en este contexto es elemental situar la unidad territorial en el marco de las posibles macrounidades que la contienen, y también respecto de las subunidades que posee. $\mathrm{Y}$ en tercer lugar se encuentra la relación, la cual deriva de 
las interacciones al interior de la escala que la constituyen dinámicamente como unidad, entegándole cierto grado de autonomía frente a otras. La dimensión de relación entrega la posibilidad de que cada escala pueda ser tratada como una totalidad, en tanto en su interior se da un conjunto de relaciones que la hacen relevante en términos socioespaciales. Esto es complejo en términos de relaciones entre escalas, dado que aquellas que se dan al interior de una escala pueden estar influenciadas por otra escala, o bien no. No hay un esencialismo relacional que permita definir influencias entre escalas de forma arbitraria (Howitt, 1998; 2003). En efecto, la definición de glocalización puede representar una forma de relación entre global y local (Swyngedouw, 1997), pero esto no es definitivo en ningún caso.

Las tres dimensiones señaladas sirven de base a algunas reflexiones en materia de gobiernos locales y de las unidades administrativas que les son asignadas. En primer lugar, los problemas planteados por múltiples autores respecto a las dinámicas espaciales y su desfase con los gobiernos locales (Berdegué et al., 2011; Fuentes et al., 2007; Heinrichs, Nuissl \& Rodríguez, 2009; Orellana, Allard, Néspolo \& Mercado, 2012) dan cuenta de una escasa reflexión en cuanto al tamaño de la escala del gobierno local. Es decir, los gobiernos locales en esos casos parecieran no adecuarse a la extensión espacial de los fenómenos sociales a los que se ven enfrentados, lo cual es particularmente explícito en el caso de las conurbaciones.

Además, específicamente en materia de relaciones, se pueden plantear dos reflexiones. Por un lado, las unidades administrativas tienden a carecer de instrumentos que les permitan articularse con otras unidades, por lo cual es difícil que logren operar relacionalmente con otras escalas. La administración regional o las asociaciones municipales apuntan en esa dirección; no obstante, no cuentan con una presencia lo suficientemente fuerte que permita hacer efectiva la articulación, siendo las unidades metropolitanas un ejemplo de esto. Por otro lado, tampoco existe mayor reflexión respecto de si las unidades administrativas poseen una coherencia interna acorde con los límites definidos. En ese plano es fundamental avanzar hacia enfoques que permitan comprender cómo la escala político-administrativa es construida socialmente, y de ese modo lograr que el nivel del gobierno local sea lo más próximo posible a las prácticas sociales. Kaiser y Nikiforova (2008) abordan la performatividad de la escala para examinar su construcción social, y con tal fin analizan la escala desde las prácticas y los discursos de los habitantes, y cómo a través de tales ejercicios producen una significación escalar. Una de las principales contribuciones de su enfoque se basa en su búsqueda de la forma en que la escala habla, lo cual se encuentra arraigado en los procesos de identificación y en el place-making, de modo que se puede identificar tanto la forma en que la escala es construida, como las fisuras y desfases entre distintas epistemologías escalares. Lo útil e interesante de esta aproximación es que invita a comprender la construcción de la escala desde las relaciones internas que se dan en ella y, por ende, desde los actores que la producen de forma dinámica. 


\section{Intermediación y gobiernos locales: la dificultad de delimitar la ciudad intermedia}

La problemática planteada en el apartado anterior se centra en la necesidad de reflexionar respecto a los procesos espaciales que acontecen en las unidades administrativas de los gobiernos locales, o bien sobre la escala del gobierno local como tal. Ahora es necesario contextualizar de un modo general la forma en que se dan las relaciones espaciales en las ciudades intermedias, entre las cuales se encuentran los casos de estudio del presente trabajo. Los procesos de intermediación, característicos de este tipo de ciudades, dan cuenta de la compleja forma en que se desenvuelven las relaciones al interior de cada ciudad, comuna y entre ellas, y representan una forma elemental de vinculación al interior de estas posibles escalas. Ilustran cómo las comunas, y los gobiernos locales asociados, se ven enfrentados a flujos e interacciones que hacen necesario comprender este tipo de asentamientos y municipios más allá de sus fronteras observables. Sin duda, la intermediación es un elemento fundamental para comprender la construcción social de las escalas con las que interactúan los gobiernos locales de ciudades intermedias.

La idea de intermediación en materia de asentamientos humanos proviene del giro conceptual desde la noción de ciudad media a la de ciudad intermedia. Este giro se asocia al abandono del criterio demográfico para definir aquellas ciudades y diferenciarlas tanto de las ciudades metropolitanas como de los asentamientos urbanos menores (Jordan \& Simioni, 1998). Este criterio ha sido cuestionado por lo estático del análisis, y porque no aborda las dinámicas y funciones que tienen las ciudades de naturaleza media en materia de articulación con asentamientos menores, así como también con áreas metropolitanas mayores (Bolay \& Rabinovich, 2004; Canziani \& Schejtman, 2013).

La respuesta al anterior planteamiento es la noción de ciudades intermedias, dado que asienta el énfasis en los procesos de acción, intercambio y apertura hacia el exterior que este tipo de ciudades desarrolla (Bolay \& Rabinovich, 2004), lo cual es coincidente con la idea de relación asociada a escala. Son los procesos de interacción con el exterior los que definen la condición de intermediación de estas ciudades y, por ende, dan origen a su denominación de ciudad intermedia. Estos procesos les entregan un protagonismo importante a los asentamientos de este tipo, en tanto operan como nodos o núcleos catalizadores de los procesos en red que se dan entre zonas rurales y asentamientos urbanos mayores, y con dinámicas económicas a nivel global. En efecto, al observar la totalidad del sistema metropolitano global, se entiende que las ciudades intermedias operan en un primer o segundo nivel de articulación entre las zonas rurales y la red global de producción (Canziani \& Schejtman, 2013).

Si bien no se puede establecer arbitrariamente el nivel en que operan las ciudades intermedias en la red urbana global, dado que ello depende del contexto en que se desarrollan e interaccionan, sí existen definiciones respecto a las relaciones en que se puede producir la intermediación. Bellet (2009) define tres escalas o formas de intermediación de las ciudades intermedias: (i) con su entorno inmediato, entendiéndolo fundamentalmente como el entorno rural al cual se circunscriben; (ii) con 
otros asentamientos urbanos o metropolitanos; y (iii) con las dinámicas nacionales e internacionales propias del contexto actual. Tal como se planteó en el párrafo anterior, las dinámicas de intermediación de estos asentamientos no son solo entre asentamientos próximos, sino también frente a flujos que exceden la escala próxima.

El problema de definir la ciudad intermedia en su totalidad por los procesos de intermediación consiste en que prácticamente cualquier asentamiento, o inclusive ser humano, se expone a procesos de interacción con el exterior. Es decir, una escasa reflexión teórica respecto a la condición de intermediación nos podría llevar a afirmar, como lo hacen Bolay y Rabinovich (2004), que toda ciudad es intermedia. Bellet (2009) plantea que la ciudad intermedia se encuentra entre la pequeña y la gran ciudad, y se caracteriza por su intermediación con diferentes escalas geográficas, que van desde lo local a lo global. Si bien a partir de la lectura de los trabajos de Bellet, así como de la literatura de ciudades intermedias en general, se logra comprender adecuadamente a qué se refiere con los procesos de intermediación para este tipo de asentamientos, efectivamente es necesaria una definición más precisa para enmarcar el debate.

Definir intermediación solo como proceso sería un esfuerzo que nos alejaría del objetivo de su delimitación territorial. El punto es aclarar el proceso de intermediación para las ciudades o nodos urbanos, y de una forma suficientemente operativa para distinguirlos de lo metropolitano y lo pequeño en términos de asentamientos, para de ese modo comprender de modo general las dinámicas territoriales. En ese plano, y a partir de las definiciones de Bellet y Llop (2004), Berdegué et al. (2011) y Canziani y Schejtman (2013), en el presente trabajo se entiende que las ciudades intermedias se caracterizan fundamentalmente por generar relaciones de interacción socioculturales, económicas y ecológicas con diferentes escalas territoriales, pero que alcanzan una intensidad y fluidez bidireccional con su escala inmediata o territorio al que se circunscriben. Esta condición las determina como funcionales de cada territorio, y prácticamente define que tengan una relación de mutua dependencia entre su escala inmediata y el nodo urbano que las constituye, pero sin dejar de interactuar con otras escalas con una menor intensidad y fluidez. Esta interacción bidireccional con la escala inmediata, que las constituye como dependientes de ese proceso, es un elemento para distinguir las ciudades intermedias de las ciudades mayores o de las metrópolis. Sin embargo, para distinguir las ciudades intermedias de las ciudades menores mediante este criterio de mutua dependencia, el criterio demográfico puede ser fundamental, en tanto en los asentamientos menores también se generan dinámicas de menor intensidad.

Definir la ciudad intermedia y abordarla en términos metodológicos a partir de las interacciones con otros asentamientos resulta sumamente complejo. En efecto, si bien gran parte de los trabajos que atienden las ciudades intermedias las definen respecto a su intermediación, en la práctica los criterios de definición tienden a basarse en una caracterización de estas en diferentes aspectos (Arenas, González \& Aliaga, 2009; Canales \& Canales, 2013; ${ }^{1}$ Maturana, 2015) -sobre todos

1 Los autores aluden más precisamente a la discusión de agrópolis, pero en la práctica son ciudades intermedias. 
demográficos-, pero poco sobre los procesos de interacción. Avances metodológicos y empíricos en materia de intermediación para el caso chileno se pueden encontrar en los trabajos de Berdegué et al. (2011), Lufin y Atienza (2015) y Mac-Clure y Calvo (2013), que se centran en las conmutaciones laborales entre comunas, pero con alcances metodológicos diferentes, y siempre desde el punto de vista cuantitativo. Lo valioso de estos trabajos es que dan cuenta de procesos de interacción efectiva entre comunas, con lo cual es posible dilucidar la intensidad de interacción en el aspecto laboral, y definir niveles de dependencia en ese aspecto. Sin embargo, la escasez de información respecto a conmutaciones o interacción entre ciudades o comunas hace necesario buscar métodos complementarios para abordar la complejidad de la intermediación en este tipo de asentamientos.

Como se mencionó anteriormente, son múltiples los trabajos que han dado cuenta de las dificultades de los gobiernos locales producto del desfase entre sus atribuciones y las dinámicas que se dan en el territorio. En materia de territorios funcionales, Berdegué et al. (2011) se refieren, entre sus recomendaciones, a la necesidad de reconocer la existencia de las ciudades que articulan territorios, que llevaría a focalizar mejor la inversión pública. Con ello los autores apuntan a la relevancia de estudiar los territorios desde un punto de vista funcional en materia de política pública, puesto que esta debe estar enfocada en las dinámicas sociales que se manifiestan en el territorio, y no en criterios arbitrarios que no dan cuenta necesariamente de lo que acontece en el plano local. Proponen al respecto fomentar las asociaciones municipales para que los gobiernos locales puedan articularse y hacer frente de mejor manera a los desafíos que supone la articulación funcional. De este planteamiento se puede deducir que es necesaria la revisión del tamaño y nivel de la escala de los gobiernos municipales, la cual se realiza a partir de un examen de las relaciones socioespaciales. Ello reafirma la necesidad de reflexionar respecto a los procesos territoriales sobre los cuales va a trabajar el gobierno local, los cuales pueden ser articulados mediante la escala.

\section{Metodología}

Ante el desafío de abordar la construcción social de la escala, tarea de especial complejidad cuando se trata de ciudades intermedias, el presente trabajo emplea tanto análisis cualitativo como cuantitativo, siendo el primero el que estructura y guía la investigación. La necesidad de comprender las prácticas espaciales y los procesos de intermediación se materializó en una investigación de tres años, a lo largo de la cual se realizaron 56 entrevistas semiestructuradas en Angol, Villarrica, Victoria y Temuco-Padre Las Casas. Las entrevistas se hicieron a actores claves de los sectores público y privado y de la sociedad civil, y se orientaron a identificar y profundizar las problemáticas político-administrativas asociadas a las dinámicas territoriales en el contexto actual de transformación, ante lo cual surgió la problemática de las atribuciones del gobierno local frente a la escala de las ciudades intermedias en cuestión. Las entrevistas se complementaron con caminatas por las ciudades con los actores claves, tal como recientes desarrollos metodológicos lo han sugerido 
(Anderson, 2004; Evans \& Jones, 2011), las que permitieron situar espacialmente los procesos de intermediación en el paisaje local.

Respecto al trabajo cuantitativo, este se centró en la metodología elaborada por Lufin y Atienza (2015) a partir de la encuesta de Caracterización Socio-económica Nacional $\left(\right.$ CASEN $\left.^{2}\right)$ de 2009, la cual evalúa la conmutación entre comunas por motivos de trabajo. Para el presente trabajo se consideró tanto a los conmutantes laborales como a los estudiantes, en tanto el objetivo es analizar el sistema en su totalidad, y la pregunta en la encuesta los considera a ambos. Si bien se desarrolló la totalidad de los índices propuestos por los autores, para el presente trabajo los elementales son el índice de retención (Eii\%), el índice de atracción bruta neta y los índices de concentración de destinos y de origen. El primero representa al porcentaje de la población que no se desplaza a otras comunas a estudiar ni trabajar, y se obtiene calculando porcentajes de la pregunta Tio de CASEN. ${ }^{3} \mathrm{El}$ segundo representa la relación entre la cantidad de personas que salen a trabajar de la comuna y las que vienen a trabajar a ella, respecto del total de ocupados y estudiantes de cada comuna. Los índices de concentración de destinos se calculan a partir de la distribución de los conmutantes de salida en distintas comunas aplicando el índice Herfindahl-Hirschman ( $\mathrm{HH})$, donde los valores más altos representan mayor diversificación. El índice de concentración de origen es lo mismo, pero evalúa la receptación de conmutantes. Además se analizaron secundariamente las preguntas de CASEN respecto al mercado de trabajo, para caracterizar de forma genérica los asentamientos más activos de la región.

Además de lo anterior, se elaboró una gráfica basada en los flujos de salida y entrada de conmutantes entre comunas de la Región de la Araucanía, donde se consideraron comunas que tuviesen una población de estudiantes y ocupados superior a 4.000, y presentaran flujos hacia un destino superiores a 200 personas.

\section{Intermediación en la Región de la Araucanía}

La Región de la Araucanía cuenta con 32 comunas que se organizan en dos provincias, siendo Angol la capital provincial de Malleco, y Temuco la de Cautín (figura 1). Respecto a los aspectos demográficos, en términos absolutos se puede afirmar que el Gran Temuco (que considera las comunas de Temuco y Padre Las Casas), Angol y Villarrica son los centros de mayor peso en la región (tabla 1), y que también cuentan con una mayor población de estudiantes y trabajadores.

2 Para la Encuesta de Caracterización Socio-económica Nacional (CASEN) 2009, la totalidad de las comunas en estudio tienen representatividad comunal (autorrepresentadas).

3 “En qué otra comuna o país trabaja o estudia?” 
FIGURA I | Centros poblados, provincias y comunas de la Región de la Araucanía

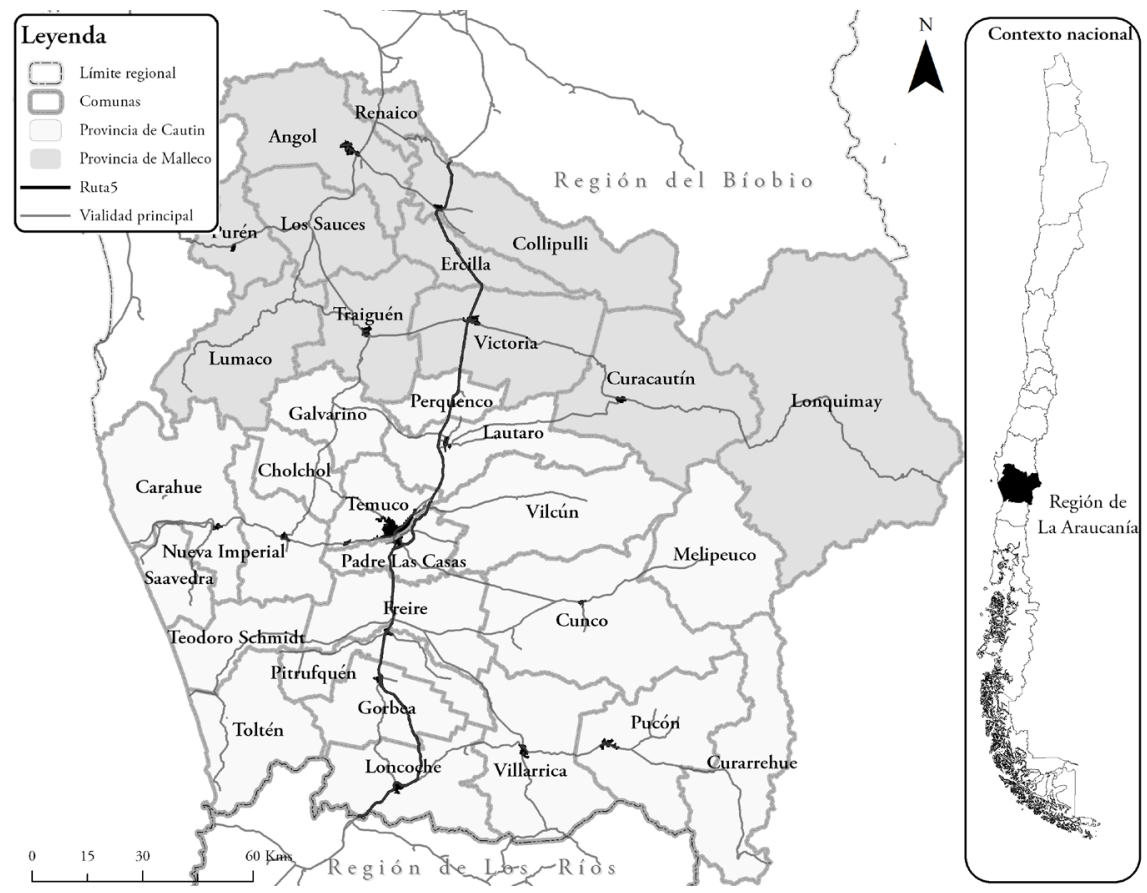

FUENTE ELABORACIÓN PROPIA

El Gran Temuco, Angol y Villarrica actúan como receptores de parte importante de los ocupados y estudiantes de las comunas aledañas. El índice de retención (Eii\% en tabla 1) da cuenta de que parte importante de las comunas de la región retienen prácticamente menos de tres cuartas partes de su población funcional. Este movimiento permite identificar algunas aristas respecto a cómo la población vive el día a día respecto a sus actividades funcionales, lo que sin duda lleva a identificar cómo se producen las relaciones al interior y entre escalas. En efecto, esto se materializa en el relato de los actores locales respecto a las ciudades de estudio y sus territorios, dado que aparecen asociaciones espaciales que se corresponden con fuertes interacciones intercomunales en la región, las cuales se articulan en los centros urbanos. 
TABLA I | Ocupados, estudiantes y conmutación por comuna

\begin{tabular}{|c|c|c|c|c|c|c|c|}
\hline & $\begin{array}{c}\text { POBLACIÓN } \\
\text { TOTAL }\end{array}$ & $\begin{array}{c}\text { OCUPADOS } \\
\text { Y ESTU- } \\
\text { DIANTES }\end{array}$ & \begin{tabular}{|c|} 
OCUPADOS Y \\
ESTUDIANTES \\
DENTRO DEL \\
TOTAL $(\%)$
\end{tabular} & $\begin{array}{c}\text { ÍNDICE DE } \\
\text { RETENCIÓN } \\
(\text { EII\%) }\end{array}$ & $\begin{array}{c}\text { ÍNDICE DE } \\
\text { ATRACCIÓN } \\
\text { NETA }\end{array}$ & \begin{tabular}{|c|} 
ÍNDICE DE \\
CONCEN- \\
TRACIÓN DE \\
ORIGEN \\
\end{tabular} & \begin{tabular}{|} 
ÍNDICE DE \\
CONCEN- \\
TRACIÓN \\
DE SALIDA
\end{tabular} \\
\hline Temuco & 236.416 & 160.244 & 67,78 & 92,09 & 14,13 & 0,09 & 0,12 \\
\hline Carahue & 20.159 & 11.317 & 56,14 & 82,12 & $-10,43$ & 0,47 & 0,27 \\
\hline Cunco & 15.414 & 7.345 & 47,65 & 76,94 & $-13,79$ & 0,36 & 0,11 \\
\hline Curarrehue & 5.599 & 3.813 & 68,10 & 89,69 & $-1,52$ & 0,3 & 0,5 \\
\hline Freire & 20.595 & 11.184 & 54,30 & 65,55 & $-28,93$ & 0,35 & 0,26 \\
\hline Galvarino & 8.591 & 4.901 & 57,05 & 85,68 & $-1,53$ & 0,3 & 0,61 \\
\hline Gorbea & 12.708 & 6.560 & 51,62 & 71,59 & $-22,97$ & 0,28 & 0,42 \\
\hline Lautaro & 27.487 & 14.587 & 53,07 & 79,87 & $-11,26$ & 0,74 & 0,32 \\
\hline Loncoche & 16.641 & 10.731 & 64,49 & 85,87 & $-6,26$ & 0,15 & 0,24 \\
\hline Melipeuco & 4.489 & 2.123 & 47,29 & 78,85 & $-14,88$ & 0,23 & 0,25 \\
\hline Nueva Imperial & 24.180 & 15.853 & 65,56 & 82,68 & $-2,10$ & 0,32 & 0,56 \\
\hline Padre Las Casas & 52.373 & 27.607 & 52,71 & 53,66 & $-39,27$ & 0,71 & 0,35 \\
\hline Perquenco & 5.261 & 2.390 & 45,43 & 76,53 & $-10,46$ & 0,28 & 0,36 \\
\hline Pitrufquén & 18.941 & 11.814 & 62,37 & 84,05 & $-2,18$ & 0,24 & 0,27 \\
\hline Pucón & 22.955 & 17.519 & 76,32 & 93,37 & $-1,20$ & 0,21 & 0,21 \\
\hline Saavedra & 10.791 & 6.252 & 57,94 & 85,72 & $-10,59$ & 0,19 & 0,17 \\
\hline Teodoro Schmidt & 12.705 & 8.146 & 64,12 & 83,64 & $-14,88$ & 0,12 & 0,25 \\
\hline Toltén & 8.242 & 4.219 & 51,19 & 78,74 & $-18,13$ & 0,16 & 0,31 \\
\hline Vilcún & 17.608 & 9.072 & 51,52 & 54,34 & $-12,76$ & 0,69 & 0,38 \\
\hline Villarrica & 43.446 & 28.825 & 66,35 & 89,45 & $-3,81$ & 0,25 & 0,13 \\
\hline Cholchol & 8.623 & 5.051 & 58,58 & 84,08 & $-11,19$ & 0,39 & 0,28 \\
\hline Angol & 39.908 & 26.452 & 66,28 & 85,88 & $-2,12$ & 0,16 & 0,1 \\
\hline Collipulli & 17.031 & 11.454 & 67,25 & 89,73 & 1,48 & 0,13 & 0,35 \\
\hline Curacautín & 12.247 & 6.070 & 49,56 & 85,24 & $-13,97$ & 0,17 & 0,51 \\
\hline Ercilla & 7.078 & 4.480 & 63,29 & 81,12 & $-12,50$ & 0,17 & 0,37 \\
\hline Lonquimay & 8.659 & 4.014 & 46,36 & 81,76 & $-16,84$ & 0,3 & 0,34 \\
\hline Los Sauces & 5.127 & 2.852 & 55,63 & 84,36 & $-4,14$ & 0,23 & 0,6 \\
\hline Lumaco & 7.690 & 4.767 & 61,99 & 89,83 & $-6,46$ & 0,17 & 0,3 \\
\hline Purén & 9.120 & 5.230 & 57,35 & 86,69 & $-7,86$ & 0,13 & 0,37 \\
\hline Renaico & 7.176 & 4.116 & 57,36 & 67,57 & $-29,15$ & 0,26 & 0,61 \\
\hline Traiguén & 13.288 & 7.505 & 56,48 & 91,35 & $-4,58$ & 0,27 & 0,16 \\
\hline Victoria & 25.469 & 14.341 & 56,31 & 86,33 & $-1,62$ & 0,16 & 0,08 \\
\hline
\end{tabular}

FUENTE ELABORACIÓN PROPIA A PARTIR DE CASEN 2009 
Respecto a las relaciones espaciales al interior de la escala regional, o bien entre unidades comunales, Temuco representa un núcleo urbano que ejerce presión sobre su territorio próximo. En este sentido, parte importante de las comunas que presentan un índice de retención bajo tienden a tener salidas de trabajadores y estudiantes muy fuertes hacia Temuco; es el caso de Padre Las Casas, Vilcún, Freire, Lautaro y Gorbea (figura 2). Estas comunas, junto con otras de menor magnitud, configuran lo que sería el núcleo funcional de Temuco, en tanto aportan parte importante de los trabajadores y estudiantes que se desenvuelven durante el día en la capital regional, y posteriormente pernoctan en ellas. Tal característica se condice con el hecho de que las comunas adyacentes a Temuco tienden a tener muy concentrados sus destinos de salida (índice de concentración de destino mayor a 0,3 en tabla 1), lo que da cuenta de que los destinos de las salidas de esas comunas se encuentran poco diversificados, en tanto la mayor parte de sus conmutantes tienden a dirigirse a Temuco. Por su parte, Angol, Victoria y Villarrica tienen más diversificadas las comunas que les aportan los conmutantes (índice de concentración de origen menor a 0,3 en tabla 1). Ello refleja que estas son las ciudades que poseen más autonomía en términos funcionales, y son atractivas como foco laboral para la población de otras comunas. Es decir, estas ciudades son las que desempeńan el principal rol de intermediación en la región, tras la capital regional.

En consideración de lo anterior, la figura 2 da cuenta de los importantes flujos de ocupados y estudiantes hacia Temuco, los cuales tienden a ser mayores en áreas de mayor proximidad a la capital regional, y también desde los principales centros poblados de la región (Angol y Villarrica). Este flujo tiende a ser bidireccional, pero de magnitud diferenciada, en tanto que si bien la población de comunas de menor peso suele movilizarse hacia Temuco, también parte de la población de Temuco lo hace hacia esas comunas, pero en menor medida. Ejemplos de ello son Vilcún, Padre Las Casas, Galvarino, Lautaro, Carahue, e incluso Villarrica. En ese sentido, Temuco recibe un importante influjo desde las comunas contiguas de Padre Las Casas y Vilcún, así como desde Lautaro.

Fuera del protagonismo de Temuco, las comunas de Villarrica, Angol y Victoria tienen una alta presencia en la región. Señal de ello es que poseen un balance neto entre conmutantes de salida y de entrada cercano a cero, como muestra el índice de atracción neta. Ello lo logran en la región solo algunas comunas con una base demográfica muy reducida, puesto que la mayoría cuenta con una mayor cantidad de conmutantes de salida.

Después de Temuco, el principal núcleo urbano es Angol, que en su condición de principal ciudad de la provincia de Malleco articula una importante cantidad de núcleos menores, como Purén, Collipulli y Renaico. Además, desde Angol también existe un flujo de salida importante hacia Los Sauces y Collipulli, lo cual se asocia a plantas de procesamiento forestal. Además, en Angol la actividad agroexportadora es sumamente relevante en términos de mercado de trabajo, pues emplea fundamentalmente población urbana de Angol y de comunas aledańas. En términos de servicios, Angol también es protagónica al ser capital provincial, con lo cual concentra diversos servicios públicos. Otro núcleo importante es Villarrica, que muestra una activa interacción bidereccional con Loncoche, junto con conmuntación de salida 
hacia Pucón. En términos productivos, Villarrica presenta una importante diversificación del mercado de trabajo asociada a la actividad forestal y agroexportadora y al sector turismo, junto con un activo sector de construcción. Además, por su relevancia demográfica frente a las comunas cercanas posee una mayor diversificación comercial, que la convierte en un polo mercantil. Finalmente, en menor escala, la comuna de Victoria tiende a articular comunas de menor peso demográfico que se encuentran en la provincia de Malleco y que se emplazan relativamente alejadas tanto de Temuco como de Angol. Si bien la actividad agrícola tiene una relevancia marcada en el mercado de trabajo comunal, el rol de la ciudad de Victoria como núcleo de servicios y comercio para sus comunas cercanas es clave.

FIGURA 2 | Flujos de conmutación entre las comunas de la Región de la Araucanía
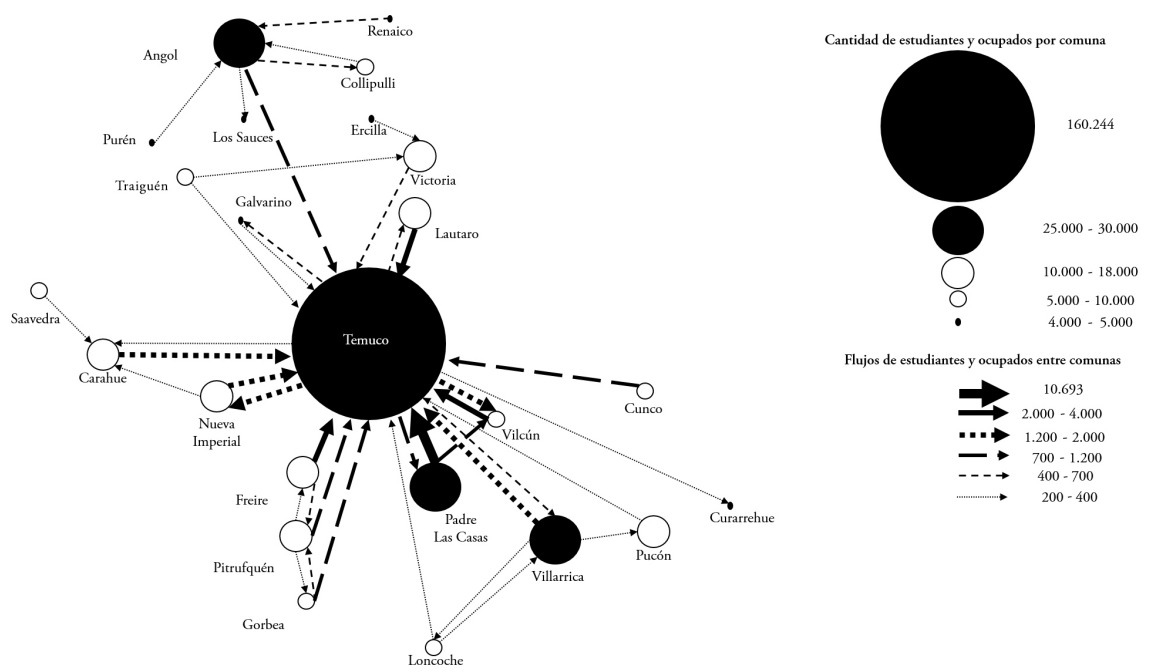

FUENTE ELABORACIÓN PROPIA

Los actores locales reconocen los procesos de interacción de población entre distintas comunas señalados y afirman que se van consolidando ciertos nodos de relaciones, los cuales van adquiriendo mayor protagonismo. De este modo, al pensar de manera comparativa, se perciben diferentes grados de autonomía entre las comunas, lo que conlleva cierto reconocimiento de diferentes niveles jerárquicos asociados a unidades comunales, o bien a asentamientos urbanos. Esto da cuenta de que si bien las prácticas espaciales construyen diferentes escalas, las comunas como tales son constitutivas del discurso de los actores. Ejemplo de este nivel jerárquico es el caso de Villarrica, donde en el último tiempo se ha generado un mayor grado de autonomía en materia de relaciones entre asentamientos, tal como lo señala el siguiente entrevistado:

Bueno, yo creo que se están consolidando como ciudades mucho más fuertes, se están como independizando un poco de la cabecera regional. Yo veo que han tenido 
un buen proceso de equipamiento (...). Temuco se convierte como una necesidad, en este caso, tal vez comercial, tal vez de servicios, pero ellos pueden vivir absolutamente independientes de este centro urbano... pensando en Villarrica. (Funcionario gobierno central)

Lo planteado hasta acá da cuenta de un sistema regional sumamente diverso respecto a las dinámicas de sus asentamientos, los que viven procesos cotidianos diferenciados en cuanto a su interacción con Temuco, como también es variada la interacción que desarrollan con otras comunas de menor peso demográfico relativo. En estas circunstancias, se hace necesario evaluar y comprender en qué medida los actores de los distintos asentamientos de mayor relevancia regional, además de Temuco, cuentan o no con las capacidades políticas suficientes para enfrentar los desafíos de su situación.

\section{Desfases escalares en las ciudades intermedias de la Región de la Araucanía}

En la Región de la Araucanía existen prácticas de movilidad funcional que dan cuenta de la conformación de ciudades intermedias que se posicionan en un nivel relacional superior a la media regional, las mismas que presentan una serie de problemáticas de índole política y administrativa asociadas a las dinámicas territoriales locales a las cuales se enfrentan. El presente análisis se centra fundamentalmente en las atribuciones de los gobiernos locales para lidiar con procesos asociados a los efectos de la intermediación, los cuales se dan con un alto grado de dinamismo. Desde la óptica de actores entrevistados, este proceso presenta un carácter dual: por un lado está la percepción de la ciudad como un nodo y red territorial bien establecido y con un cierto nivel de autonomía; por otro, se percibe la falta de herramientas para abordar política y administrativamente los desafíos de dicha condición. Tanto en términos de tamaño como de relaciones, esto sin duda representa un problema de escala, el cual también posee una impronta asociada al cambio de nivel.

Los actores entrevistados reconocen explícitamente la dificultad de hacer frente a los cambios en materia de relación escalar, que se materializan fundamentalmente en transformaciones urbanas. Afirman que los gobiernos locales no cuentan con las herramientas necesarias para abordar estos cambios, tanto desde el punto de vista técnico como del relativo a las atribuciones legales. Además de la carencia de atribuciones de parte del gobierno local para planificar y regular el crecimiento urbano de su propio asentamiento, se plantea un desfase relacional entre escalas. Esto no solo por la desarticulación entre el gobierno local y las instituciones centrales, sino también porque ello implica una falta de consideración de las perspectivas de los actores locales.

Los principales problemas de intermediación que enfrentan los gobiernos locales en materia de desarrollo urbano pasan por la vialidad y la expansión del área urbana en general. Se suma a estas perspectivas la invisibilidad política relacionada con el carácter intercultural de este tipo de asentamientos, presente sobre todo en el rol que juegan respecto al territorio rural, donde existe una presencia mayor de población mapuche. Tal como se describe en la tabla 2, es en función de estas problemáticas 
urbano-territoriales, reconocidas y jerarquizadas por los actores locales, que se genera un análisis de los procesos de intermediación como un fenómeno de escala en sus dimensiones de tamaño, nivel y relación. A continuación se desarrollan cada una de estas problemáticas con mayor detención.

TABLA 2 | Problemática escalares de los gobiernos locales de las ciudades intermedias

\begin{tabular}{|l|l|l|l|}
\cline { 2 - 4 } \multicolumn{1}{c|}{} & \multicolumn{1}{c|}{ TAMAÑo } & \multicolumn{1}{c|}{ NIVEL } & \multicolumn{1}{c|}{ RELACIón } \\
\hline Vialidad & $\begin{array}{l}\text { Movilidad en ciudades } \\
\text { intermedias trasciende } \\
\text { límites comunales, y los } \\
\text { proyectos de vialidad son } \\
\text { pensados en otro tamaño } \\
\text { relacional. }\end{array}$ & $\begin{array}{l}\text { Las decisiones en la materia } \\
\text { pasan por el nivel del } \\
\text { gobierno central, al igual } \\
\text { que el presupuesto. El } \\
\text { nivel del gobierno local es } \\
\text { relativamente obviado. }\end{array}$ & $\begin{array}{l}\text { Se da una relación impo- } \\
\text { sitiva desde la escala del } \\
\text { gobierno central hacia el } \\
\text { gobierno local. }\end{array}$ \\
\hline $\begin{array}{l}\text { Crecimiento } \\
\text { urbano }\end{array}$ & $\begin{array}{l}\text { Cambios en la dimensión } \\
\text { del área urbana. }\end{array}$ & $\begin{array}{l}\text { Normativa definida a } \\
\text { nivel nacional, con escasa } \\
\text { consideración de lo que } \\
\text { sucede en el nivel comunal } \\
\text { de ciudades intermedias. }\end{array}$ & $\begin{array}{l}\text { 1) Relación pasiva desde la } \\
\text { escala del gobierno central } \\
\text { hacia el gobierno local. } \\
\text { 2) Falta de instrumentos } \\
\text { para que el gobierno local } \\
\text { injiera en las dinámicas } \\
\text { territoriales. }\end{array}$ \\
\hline Interculturalidad & - & $\begin{array}{l}\text { Las dinámicas de inter- } \\
\text { culturalidad en ciudades } \\
\text { intermedias acontecen en } \\
\text { un nivel no percibido por } \\
\text { los gobiernos locales. }\end{array}$ & $\begin{array}{l}\text { Relación entre dinámicas } \\
\text { territoriales de ciudades } \\
\text { intermedias y gobiernos } \\
\text { locales en materia de inter- } \\
\text { culturalidad tienden a ser } \\
\text { patrimoniales, u omitidas. }\end{array}$ \\
\hline
\end{tabular}

FUENTE ELABORACIÓN PROPIA

\section{Vialidad}

Esta problemática deviene de la necesidad de desarrollar mejoras viales frente a las intensas interacciones de movilidad que presentan las ciudades intermedias con el territorio en que se circunscriben, al igual que con otras escalas de mayor nivel, aspecto de gran conflictividad desde la perspectiva de los actores locales.

Considerada desde la perspectiva de escala, en materia de vialidad se pueden distinguir dos desfases. Primero, en términos de tamaño, las ciudades intermedias establecen redes de movilidad funcionales que trascienden la escala comunal, como quedó expresado empíricamente en las conmutaciones; desde la perspectiva de los actores, se aprecia que dicho desfase es fruto de la incapacidad del gobierno local para absorber la complejidad de las dinámicas de movilidad de la ciudad intermedia. Segundo, en términos de nivel y relación, el problema central radica en que el gobierno local enfrenta un desfase institucional respecto del gobierno central, el cual tiene un alto grado de injerencia en materia de vialidad.

El desfase escalar entre el gobierno central y el gobierno local puede ser ilustrado con las transformaciones viales en Villarrica, una de las ciudades que mayor crecimiento ha experimentado en el último periodo. Desde la perspectiva de los funcionarios del gobierno local, el desfase escalar se materializó en que los operarios del gobierno central no consideraron la experiencia del municipio en la localización 
del acceso a la ciudad, lo que implicó que las decisiones del gobierno central en materia de vialidad fueran unilaterales:

Con el mop [Ministerio de Obras Públicas] es compleja la relación, porque tienen una forma de actuar bastante poco participativa respecto a los municipios. Generalmente ellos actúan de modo a sus criterios y son poco abiertos a mostrar las obras que van a hacer o cómo lo van a hacer. (Funcionario municipal)

Esta lejanía institucional en el proceso de toma de decisiones se refleja también en la forma en que se van desarrollando los proyectos comandados por instituciones ministeriales, y la escasa información que manejan los municipios al respecto. Nuevamente Villarrica sirve para ejemplificar esto, en tanto las modificaciones viales al interior de la ciudad fueron decididas por el gobierno central, y se afirma que prácticamente se hicieron visibles al momento de su ejecución.

Esa obra genera un montón de conflictos porque nunca se conoció el detalle del proyecto, se fue dando cuenta de la medida que se iba ejecutando, iban apareciendo problemas. (Funcionario municipal)

Por un lado, no existen instrumentos asociados al gobierno local para hacer frente a estas dinámicas de intermediación. Por otro, el gobierno central actúa sin una consideración adecuada de las relaciones internas de la escala. Las relaciones que acontecen en la escala comunal se complejizan al momento de enmarcarse en una escala de mayor nivel en términos político-administrativos, lo que lleva a un desfase entre gobiernos locales, que deben hacer frente a una densificación en sus interacciones cotidianas (como nodos), y un gobierno central que decide arbitrariamente. Las atribuciones de las municipalidades, establecidas en la Ley Orgánica Constitucional de Municipalidades (LOCM), no consideran las herramientas necesarias para abordar procesos urbanos como los que enfrentan las ciudades intermedias, en tanto tienden a derivar la problemática al gobierno central.

\section{Crecimiento urbano}

Desde otro punto de vista, la migración desde los asentamientos rurales hacia las ciudades intermedias ha sido un proceso creciente en los últimos años, lo que se ha traducido en crecimiento de la mancha urbana. Por su parte, los procesos de conmutación asociados a una aglomeración espacial de fuentes de trabajo y servicios también potencian procesos de inmigración a estas ciudades. El dinamismo dado por las transformaciones en servicios, conectividad y mercados de trabajo, es un factor que ha incidido en esta creciente tendencia inmigratoria propia de las ciudades intermedias en Chile, las cuales presentan mayores tasas de crecimiento demográfico (Borsdorf, Marchant \& Sánchez, 2012; Canales \& Canales, 2013) que las áreas metropolitanas, y por su menor peso demográfico relativo son considerablemente más observables en el paisaje urbano.

El crecimiento urbano de las ciudades intermedias conlleva la emergencia de nuevos espacios, los cuales se asocian principalmente a la producción de vivienda para distintos niveles socioeconómicos. Estos cambios en la escala urbana dentro de la comuna traen consigo nuevas problemáticas, las cuales, a nivel de compromisos, 
buscan ser tratadas por el gobierno local. Producto de la falta de planificación se menciona la carencia de equipamiento urbano en los nuevos sectores periféricos de la ciudad, particularmente en los caracterizados por población de bajos ingresos, los cuales a su vez enfrentan procesos crecientes de segregación socioespacial. Elementos básicos, como que las calles cuenten con un diseńo apropiado para la recolección de residuos sólidos domiciliarios, infraestructura de esparcimiento y conectividad apropiada a la ciudad, son los principales puntos que intentan tratar los gobiernos locales frente al cambio del tamaño en la escala urbana.

Esta tendencia se enfrenta con un desajuste respecto al nivel en que es definida la normativa. En efecto, el principal desfase escalar de esta problemática se encuentra entre una normativa estandarizada a nivel nacional y gobiernos locales que deben hacer frente a procesos de crecimiento urbano desarrollados en su mayoría por el sector privado, pero sin atribuciones concretas para ello:

No podemos detener ese crecimiento, que la empresa hace cambio de suelo y presenta un proyecto, y si cumple con todas las normativas ambientales, en general, le aprueban el proyecto. Y después cómo se modifica... Con respecto a ese tema, no hay una norma que dé atribuciones al municipio para oponerse a un proyecto habitacional que cumpla con las normas. (Funcionario municipal)

Este desfase en materia de nivel implica necesariamente un desfase en términos relacionales. En primer lugar, si bien los gobiernos locales conocen las dinámicas y los desafíos cotidianos propios de los procesos locales, no cuentan con la capacidad suficiente para ingerir en ellos. Básicamente, el problema pasa por un diseńo estandarizado de gobiernos locales, el cual no deja espacio para que estos dispongan de mecanismos de relación con las dinámicas del territorio. Discursivamente, la estructura institucional descentralizada asigna a los municipios los compromisos, pero no la capacidad de acción y recursos necesarios para resolver los problemas territoriales.

Por otro lado, el desfase relacional entre el gobierno central y el gobierno local en materia de marco normativo es un problema medular. Se reconoce una relación pasiva desde el gobierno central hacia el gobierno local, en tanto las directrices del primero se centran en que los proyectos de vivienda se cińan a la normativa establecida, mientras observan escasamente lo que acontece en el territorio. Se trata de una normativa que no entrega elementos complementarios para que los gobiernos de unidades administrativas locales que cuentan con un mayor dinamismo demográfico y de movilidad incidan en el desarrollo local. Esto deja una cierta sensación de impotencia en los gobiernos locales, los cuales carecen de las facultades para hacer frente a los vacíos que se generan entre la norma legal y las prácticas de las inmobiliarias privadas:

La empresa compró una cantidad de hectáreas gigante en la cual tiene un plan de cumplir con 1.600 casas, pero ellos presentan proyectos de vivienda de 159 viviendas, porque la normativa dice que sobre 160 viviendas tú tienes que generar estudios viales y mejorar las vías ¿cierto? Vamos en la etapa 14 ¿y vamos a hacer un estudio? No, no puedes pedirle un estudio porque son 159 viviendas. ¿Qué hacemos nosotros, si sabemos que va a construir mil casas y el tipo las va a hacer por 
etapas para no mejorar la viabilidad? Tampoco te ayuda el Ministerio, porque te dicen "no, es por normativa, yo no les puedo pedir", y yo digo "pero si esto se está sumando”. Entonces, ¿cómo lo hacemos? (Funcionario municipal)

\section{Interculturalidad}

Respecto a las problemáticas asociadas a la intermediación en las ciudades de estudio, una de las dimensiones básicas para abordar la producción del espacio social y la multiplicidad de escalas existentes, es el carácter intercultural de la región. Las relaciones interculturales tienen una incidencia fundamental en la construcción del espacio local, fruto de las interacciones cotidianas entre las ciudades intermedias analizadas y las zonas rurales, ya que si bien históricamente las comunidades mapuche asocian su adscripción territorial a espacios rurales, ${ }^{4}$ el sistema urbano desempeña un rol crucial en las interacciones sociales.

No se puede dejar de mencionar que la dimensión intercultural asociada a la intermediación en la Araucanía debe entenderse en un marco histórico mayor, como es que las ciudades de la región emergieron en el proceso de ocupación de tierras indígenas por parte del Estado chileno. En ese contexto, en las ciudades de la Araucanía se ha desarrollado un proceso de invisibilización de la cultura mapuche, a la vez que ellas han sido el motor de la transformación territorial que ha vivido la zona desde hace varias décadas.

Sin embargo, y pese a que en La Araucanía las ciudades contienen más elementos asociados a la colonización y modernización del territorio que al origen ancestral de este, en ellas se encuentran múltiples elementos que dan cuenta de su contexto intercultural. En una aproximación a las expresiones en el paisaje de las ciudades en estudio surgen múltiples elementos que dan cuenta de la presencia mapuche en el espacio urbano a un nivel simbólico, visible por medio de la resignificación de espacios culturales o expresiones artísticas en los murales de la ciudad. Además, las prácticas comerciales -tanto formales como informales- desarrolladas desde el mundo indígena hacia estas ciudades son un elemento constitutivo de su carácter, puesto que la población mapuche suele desplazarse a los nodos urbanos para transar productos elaborados en zonas rurales. Esta dimensión se hace visible materialmente en los terminales rurales de estas ciudades.

En este marco, los procesos de intermediación asociados a la movilidad cotidiana entre las zonas urbanas y rurales también dan cuenta de dinámicas interculturales, facilitadas por el hecho de que la ciudad y la comunidad mapuche emplazada en la zona rural se encuentran a una distancia que puede cubrirse en una o dos horas de viaje (Aravena, Gissi \& Toledo, 2005). Consecuencia de ello es que la identidad de estas ciudades, basada en sus dinámicas territoriales, también corresponde a diversas relaciones interculturales que se expresan en el espacio urbano. Se trata de relaciones económicas, institucionales, sociales que se articulan en la ciudad, y

Si bien el fenómeno requiere ser analizado en una mayor profundidad al considerar el aumento de la población mapuche urbana, el componente rural de la población mapuche siguen siendo preponderante, con $70,1 \%$ del total en la Región de la Araucanía (Instituto Nacional de Estadísticas [INE], 2005). 
cuyos significados se alimentan de las interacciones del entorno inmediato con la vida cotidiana de la ciudad. Es aquí donde se reconoce el carácter intercultural de la ciudad. Tal como lo señala uno de los entrevistados:

La realidad está en el entorno, y allí está la cultura, no en lo bonito, en lo que tú haces, en la arquitectura, no, en la diversidad está la riqueza de la ciudad. (Dirigente social)

Sin embargo, las expresiones de interculturalidad señaladas operan en un nivel distinto al del gobierno local -no necesariamente en términos jerárquicos-, en tanto las relaciones cotidianas y sentidos de adscripción identitaria no aparecen destacados en las problemáticas diagnosticadas por los gobiernos locales, o bien son muchas veces localizados fuera de los límites urbanos.

En los gobiernos locales, la gestión relacionada a las dinámicas interculturales tiende a ser abordada desde una visión patrimonial, la cual reduce las relaciones que se dan en el espacio a objetos o lugares de resignificación sobre los pueblos originarios, cultivando así una visión fija de su cultura, sin lograr dar cuenta de las relaciones dinámicas que subyacen a los usos del espacio. Esta conceptualización, o más bien omisión, da cuenta del desfase escalar entre el gobierno local y las prácticas sociales que se dan en el territorio.

Desde otro punto de vista, algunos de los actores entrevistados señalan que la historia urbana $-\mathrm{y}$ con mayor fuerza las transformaciones socioeconómicas de los últimos años- han tendido a reducir la identidad intercultural fundamental de estas ciudades. En ellas, el problema radica en la falta de valoración de las identidades localmente fundadas y en la necesidad de rescatarlas. En el proceso de las entrevistas por la ciudad, muchos de los consultados dieron cuenta de la invisibilidad política de las relaciones interculturales existentes en las ciudades, ausencia patente sobre todo en la falta de consideración de las prácticas de intermediación de la población mapuche, presentes en las dinámicas de relación entre la ciudad y territorio rural. La interculturalidad es constitutiva de las prácticas sociales que se dan en la escala local, y es un elemento que no es adecuadamente reconocido por el gobierno local. Este punto es quizás el mayor desencuentro entre la escala del gobierno local y la de las prácticas sociales, y el buen ejercicio del primero depende de lo segundo.

\section{Conclusiones}

Este trabajo ha abordado la relación existente entre los procesos socioespaciales que se dan en las ciudades intermedias de la Región de La Araucanía y los gobiernos locales, principalmente en el ámbito de la planificación urbano-territorial. Para comprender la complejidad de esta relación y las problemáticas que derivan de ella, este trabajo se ha centrado en los conceptos de escala e intermediación, los que, si bien han cobrado relevancia por separado en los estudios urbanos durante últimos años, no se habían integrado teóricamente hasta ahora. Esto ha permitido identificar y discutir de manera más precisa diversos desfases escalares existentes entre los fenómenos de intermediación propios de las ciudades estudiadas y las capacidades de los gobiernos locales para atenderlas. En efecto, se han distinguido tres escalas diferentes. En la base se encuentran las dinámicas locales, las cuales se arraigan en 
los procesos de intermediación y en las particularidades territoriales; en segundo lugar se encuentra el gobierno local, el cual no necesariamente posee el mismo tamańo que el nivel de las relaciones locales, pero sí tiene un mayor grado de relación con estas; y en el nivel más alto se encuentra el gobierno central, el cual no reconoce los tamaños de las otras dos escalas de menor nivel, y actúa con autoridad en materia relacional frente a ellas.

De este modo, los resultados expuestos permiten rescatar dos elementos fundamentales para la planificación sustentable de la ciudad intermedia y el rol que en ella tienen los gobiernos locales.

En primer lugar, los gobiernos locales no logran atender de forma apropiada la complejidad presente en los procesos de intermediación. Por un lado, se ha argumentado tanto teórica como empíricamente que las ciudades intermedias se constituyen como complejas sinapsis urbano-territoriales, construidas dinámicamente por interacciones sociales en (y entre) diversas escalas. En el caso del sistema urbano de la Región de La Araucanía, esto se demuestra en parte por la existencia de fuertes dinámicas de conmutación en estudiantes y ocupados, proceso en que las ciudades de Temuco-Padre las Casas, Angol y Villarrica emergen como nodos bastante autónomos, y a la vez como articuladores de una red de interacción urbana-territorial. Por otro lado, estos procesos de intermediación socioespacial de carácter dinámico no solo contrastan con la ontología de unidades administrativas locales fijas (como son las comunas), sino también traspasan sistemáticamente la capacidad y herramientas de que disponen los gobiernos locales para atender diversas problemáticas territoriales.

Segundo, si bien la ciudad intermedia genera redes de intermediación interescalar, es la relación con su territorio circundante (o hinterland)-además del tamaño espacial y demográfico- lo que le da realmente su carácter diferenciador respecto a otros tipos de asentamientos urbanos (menores y metropolitanos). De este modo, si bien ciertas problemáticas emergentes en torno a vialidad, crecimiento urbano e interculturalidad están presentes también en las ciudades metropolitanas, sería un error reducir el análisis de las ciudades intermedias al hacerlas un espejo de sus pares metropolitanas. Por el contrario, en el caso de las ciudades intermedias estas problemáticas tienen rasgos relativamente únicos, debido a la interdependencia de las dinámicas urbanas con su hinterland y los desafíos que conlleva su planificación y gestión local. Así, las problemáticas organizacionales y funcionales que enfrentan los gobiernos locales de la ciudad intermedia están dadas en gran medida por la miopía imperante en los tomadores de decisiones y en las políticas de planificación, incapaces de reconocer estos procesos de intermediación como constitutivos de la ciudad intermedia. Esto se condice con lo que se introdujo en el apartado teórico, en cuanto a que la agenda de descentralización en Chile no incorpora una visión espacio-temporal donde se reconozca la particularidad de la ciudad intermedia y que, desde ahí, oriente las atribuciones y los procesos de gestión de los gobiernos locales, aspectos necesarios para atender su complejidad. Si bien la agenda de descentralización contiene elementos relacionales entre comunas, en la práctica estos se centran en problemáticas de escala metropolitana. Esto refuerza la idea de la ceguera de los tomadores de decisiones respecto a los procesos que acontecen en las ciudades de nivel intermedio. 
Ahora bien, en caso de que sean reconocidos los procesos de intermediación como constitutivos de estas ciudades, el paso siguiente es analizar técnica y políticamente la forma en que ellos son incorporados por las políticas públicas. Berdegué et al. (2011) apuntan a que las asociaciones municipales son instancias que deben ser fomentadas en términos de territorios funcionales; no obstante, no existe claridad en cuanto a los elementos concretos de planificación urbana y territorial orientados a tal fin. Los Planes Regionales de Ordenamiento Territorial (рROT), en el caso chileno, podrían ser un instrumento potente, siempre y cuando lograran ser vinculantes y permitieran planificar de forma efectiva, sin limitarse a regular aspectos urbanos de gran especificidad. Además, es fundamental hacerse cargo de los procesos de interacción entre las ciudades con su entorno circundante, de suma relevancia en el caso de las ciudades intermedias, los cuales acontecen dentro de cada comuna. Esto da cuenta de que la intermediación no solo es un problema de coordinación, sino también de atribuciones y capacidades para hacer frente a procesos de interacciones sociales en el espacio. La discusión en materia de políticas públicas sobre ciudades intermedias es un debate abierto, necesario, y que debiese ser una línea de investigación prioritaria en la agenda de descentralización.

Metodológicamente, este trabajo se ha propuesto el desafío de atender con mayor coherencia el proceso de construcción social de la escala en el contexto de las ciudades intermedias. De este modo, los actuales estudios de ciudades intermedias -que han estado principalmente comandados por metodologías cuantitativas que refieren al ámbito de tamaño demográfico y espacial- deben complementarse o bien trascender a metodologías mixtas que también incorporen las dimensiones de nivel y relación en los estudios de escala. Estos últimos contienen prácticas, discursos y significados de los habitantes en el espacio que no solo se vuelven vitales para la comprensión de la ciudad intermedia como fenómeno particular, sino también para una planificación urbana-territorial más apropiada social, ecológica y políticamente.

\section{Agradecimientos}

Esta investigación ha sido financiada por los siguientes fondos públicos: Fondecyt de Iniciación N. ${ }^{\circ} 11130519$; Proyecto Conicyt/Fondap N.o 15110020 (Cedeus); Proyecto Conicyt/Fondap N. 15110006 (CIIR). Se gradece a los pares evaluadores de esta revista por sus valiosas contribuciones.

\section{Referencias bibliográficas}

Anderson, J. (2009). Talking whilst walking: A geographical archaeology of knowledge. Area, 36(3), 254-261. https://doi.10.1111/j.0004-0894.2004.00222.x

Aravena, A., Gissi, N. \& Toledo, G. (2005). Los mapuches más allá y más acá de la frontera: Identidad étnica en las ciudades de Concepción y Temuco. Sociedad Hoy (8-9), 117132. http://www.redalyc.org/pdf/902/90221948010.pdf 
Arenas, F., González, D. \& Aliaga, G. (2009). La conurbación Rancagua-Machalí: transformaciones socioespaciales de una ciudad media. En R. Hidalgo, C. de Mattos \& F. Arenas (eds.), Chile: del país urbano al país metropolitano (pp. 347-358). Santiago, Chile: GEOlibros-Colección EURE-libros.

Bellet, C. (2009). Las ciudades medias o intermedias en un mundo globalizado. Lleida: Ediciones de la Universitat de Lleida.

Bellet, C. \& Llop, J. M. (2004). Miradas a otros espacios urbanos. Scripta Nova: Revista electrónica de geografía y ciencias sociales, 8(165). http://www.ub.edu/geocrit/sn/sn165.htm

Berdegué, J., Jara, B., Fuentealba, R., Tohá, J., Modrego, F., Schejtman, A. \& Bro, N. (2011). Territorios funcionales en Chile. Documento de Trabajo n. ${ }^{\circ}$ 102. Programa Dinámicas Territoriales Rurales. Santiago, Chile: Centro Latinoamericano para el Desarrollo Rural (Rimisp). http://bit.ly/1xqhnKV

Bolay, J.-C. \& Rabinovich, A. (2004). Intermediate cities in Latin America: Risk and opportunities of coherent urban development. Cities, 21(5), 407-421. https://doi. org/10.1016/j.cities.2004.07.007

Boisier, S. (2010). Territorio, Estado y sociedad en Chile: La dialéctica de la descentralización entre la geografia y la gobernabilidad. Santiago, Chile: Mago Editores. Tesis original en http://bit.ly/2ntlwRr

Borsdorf, A., Marchant, C. \& Sánchez, R. (2012). Changes in urbanization processes. The intermediate cities in the Chilean urban system. En D. Rivera (Ed.), Chile: Environmental, political and social issues (pp. 159-173). Nueva York: Nova Publishers.

Brenner, N. (2001). The limits to scale: Methodological reflections on scalar structuration. Progress in Human Geography, 25, 591-614. https:// doi.10.1191/030913201682688959

Canales, A. \& Canales, M. (2013). De la metropolización a las agrópolis. El nuevo poblamiento urbano en el Chile actual. Polis, 12(34), 31-56. https://doi.org/10.4067/S071865682013000100003

Canziani, J. \& Schejtman, A. (2013). Ciudades intermedias y desarrollo territorial. Lima: Centro Latinoamericano para el Desarrollo Rural (Rimisp).

Comisión Asesora Presidencial en Descentralización y Desarrollo Regional. (2014). Propuestas de Política de Estado y Agenda para la Descentralización y el Desarrollo Territorial de Chile. Hacia un país desarrollado y justo. Informe. http://tenred.cl/wp-content/ uploads/2014/10/PROPUESTAS-COMISION-DESCENTRALIZACION-FINAL. pdf

Evans, J. \& Jones, P. (2011). The walking interview: Methodology, mobility and place. Applied Geography, 31(2), 849-858. https://doi.10.1016/j.apgeog.2010.09.005

Falleti, T. (2010). Decentralization and subnational politics in Latin America. Cambridge, MA: Cambridge University Press.

Fuentes, L., Allard, P. \& Orellana, A. (2007). El municipio y la gobernabilidad del territorio comunal. En T. Matus (coord.), La reforma municipal en la mira. Identificando los municipios prioritarios en la Región Metropolitana: Complejidad comunal versus condiciones para la calidad de la gestión municipal (pp. 43-84). Santiago, Chile: Expansiva/Observatorio de Ciudades Universidad Católica, Escuela de Trabajo Social (pUC). http://www.expansiva.cl/media/archivos/20071030101546.pdf 
Heinrichs, D., Nuissl, H. \& Rodríguez, C. (2009). Dispersión urbana y nuevos desafíos para la gobernanza (metropolitana) en América Latina: el caso de Santiago de Chile, EURE, 35(104), 29-46. https://doi.org/10.4067/S0250-71612009000100002

Howit, R. (2003). Scale. En J. Agnew, K. Mitchell \& G. Toal (eds.), A companion to Political Geography (pp. 138-157). Oxford: Blackwell.

Howitt, R. (1998). Scale as relation: musical metaphors of geographical scale. Area, 30(1), 4958. https://doi.10.1111/j.1475-4762.1998.tb00047.x

Instituto Nacional de Estadísticas (INE), Chile. (2005). Estadisticas Sociales de los Pueblos Indigenas en Chile. Censo 2002. Santiago, Chile: Instituto Nacional de Estadísticas/ Ministerio de Planificación Nacional. http://bit.ly/1zwRAkl

Jordan, R \& Simioni, D (Eds.). (1998). Ciudades intermedias de América Latina y el Caribe: propuestas para la gestión urbana. [LC/L. I I 77]. Santiago, Chile: Comisión Económica para América Latina y el Caribe (CEPAL)/Ministero degli Affari Esteri - Cooperazione Italiana. http://repositorio.cepal.org/bitstream/handle/11362/31024/1/S9800066_ es.pdf

Kaiser, R. \& Nikiforova, E. (2008). The performativity of scale: The social construction of scale effects in Narva, Estonia. Environment and Planning D: Society and Space, 26(3), 537562. https://doi.10.1068/d3307

Lufin, M. \& Atienza, M. (2015). El papel de las ciudades intermedias en la red de flujos de conmutación en Chile. En F. Maturana \& A. Rojas (Eds.), Ciudades intermedias en Chile. Territorios olvidados (pp. 151-178). Santiago, Chile: RIL Editores.

Mac Clure, O. \& Calvo, R. (2013). Desigualdades sociales y tipos de territorios en Chile. Polis. Revista Latinoamericana, 12(34), 467-490. https://doi.org/10.4067/S071865682013000100023

Marston, S., Jones III, J. \& Woordward, K. (2005). Human Geography without scale. Transactions of the Institute of British Geographers, 30(4), 416-432. https://doi.10.1111/ j.1475-5661.2005.00180.x

Massey, D. (1994). Space, Place and gender. Minneapolis, mN: University of Minnesota Press.

Maturana, F. \& Rojas, A. (2015). Ciudades intermedias en Chile. Santiago, Chile: RIL Editores

Montecinos, E. (2005). Los estudios de descentralización en América Latina: una revisión sobre el estado actual de la temática, EURE, 31(93), 73-88. http://www.scielo.cl/pdf/eure/ v31n93/art05.pdf

Moore, A. (2008). Rethinking scale as a geographical category: From analysis to practice. Progress in Human Geography, 32(2), 203-225.

Orellana, A., Allard, P., Néspolo, R. \& Mercado, J. (2012). Gestión urbana municipal a escala metropolitana: modelos en competencia. Revista de Geografía Norte Grande, (51), 6780. https://doi.org/10.4067/S0718-34022012000100004

Programa de Naciones Unidas para el Desarrollo (PNUD). (2007). Lineamientos Básicos para la Descentralización Administrativa y Fiscal. Manual de Orientación. Bogotá: Universidad Externado de Colombia.

Shah, A. (2006). Local governance in developing countries. Washington D.c.: Banco Mundial.

Smith, N. (1992). Geography, difference and politics of scale. En E. Doherty, M. Graham \& M. Malek (eds.), Postmodernism and Social Sciences (pp. 57-79). Nueva York: St. Matin's Press. 
Subsecretaría de Desarrollo Regional (Subdere), Ministerio del Interior, Chile. (2007). Estudio evaluación ex-post creación de 11 comunas: 1994-2004. Informe final. Santiago de Chile: Ministerio del Interior, Departamento de Estudios y Evaluación. http://www.subdere. gov.cl/sites/default/files/documentos/articles-73814_recurso_1.pdf

Swyngedouw, E. (1997). Neither local nor global: Glocalization and the politics of scale. En K. R. Cox (Ed.), Spaces of globalization: Reasserting the power of the local (pp. 137-166). Nueva York: Guilford Press.

Vial, C. (2015). Ciudades intermedias y municipalidades: La carencia de un gobierno. En F. Maturana \& A. Rojas (eds.), Ciudades intermedias en Chile. Territorios olvidados (pp. 75-104). Santiago, Chile: RIL Editores. 